\title{
Numerical study of polymer tumbling in linear shear flows
}

\author{
A. Puliafito ${ }^{\mathrm{a}, \mathrm{c}, *}, \mathrm{~K}$. Turitsyn ${ }^{\mathrm{a}, \mathrm{b}, \mathrm{c}}$ \\ a INLN, UMR 6618 CNRS, 1361, Route des Lucioles, 06560 Valbonne, France \\ ${ }^{\mathrm{b}}$ Landau Institute for Theoretical Physics, Kosygina 2, Moscow 119334, Russia \\ c CNLS, Theoretical Division, Los Alamos National Laboratory, Los Alamos, NM 87545, USA \\ Received 18 March 2005; received in revised form 27 July 2005; accepted 27 July 2005 \\ Available online 16 August 2005 \\ Communicated by $\mathrm{M}$. Vergassola
}

\begin{abstract}
We investigate numerically the dynamics of a single polymer in a linear shear flow. The effects of thermal fluctuations and randomly fluctuating velocity gradients are both analyzed. Angular, elongation and tumbling time statistics are measured numerically. We perform analytical calculations and numerical simulations for a linear single-dumbbell polymer model comparing the results with previous theoretical and experimental studies. For thermally driven polymers the balance between relaxation and thermal fluctuations plays a fundamental role, whereas for random velocity gradients the ratio between the intensity of the random part and the mean shear is the most relevant quantity. In the low-noise limit, many universal aspects of the motion of a polymer in a shear flow can be understood in this simplified framework.
\end{abstract}

(C) 2005 Elsevier B.V. All rights reserved.

Keywords: Fluid dynamics; Single polymer dynamics

\section{Introduction}

Thanks to recent improvements in experimental techniques it is nowadays possible to follow the motion of individual molecules in solvents [1-9]. The characterization of polymer dynamics at the level of a single molecule is a first step towards the understanding of mechanical interactions between biomolecules (see, e.g. [10-17]), of the fundamental rheology of polymer solutions, and of the viscoelastic properties of more complex flows (see, for example [18], and references therein for elastic turbulence).

Recently measurements of elongation and orientation with respect to simple external flows have been performed $[7,9]$ in order to analyze how the conformation of a single molecule can be modified by an external field.

\footnotetext{
* Corresponding author. Tel.: +334929673 35; fax: +33493652517.

E-mail address: alberto.puliafito@inln.cnrs.fr (A. Puliafito).
} 
As the number of degrees of freedom needed to fully describe a macromolecule is extremely high it is necessary to formulate theoretical models, to verify them with numerical simulations, to simplify the problem, and to understand which observables play a key role.

Large numbers of papers have been written on the subject of single polymer dynamics in shear flows from experimental, numerical and theoretical viewpoint by Chu, Larson, Shaqfeh and their respective collaborators.

A polymer molecule in a plane, linear, steady shear flow is oriented in the flow direction by the velocity field. As the rotational motion of the polymer is determined only by the velocity difference in the space points, when it is aligned along the shear direction the external flow effect becomes negligible, and the thermal noise is the most relevant external force. Thermal fluctuations can push the polymer to regions where the external flow is relevant again. In these cases the shear flow can induce a fast rotation and align the polymer again along the (reverse) flow direction, i.e. the polymer tumbles [21,3,4,8,9,7].

This phenomenon can happen via several conformational pathways due to the complexity of the motion of a polymer molecule (see, for example [8,9]), and can be fully described only by taking into account all the degrees of freedom of the polymer. Unfortunately in the framework of these complex polymer models it is very difficult to obtain analytical results.

The main goal of our paper is to test numerically the predictions of recent theoretical and experimental studies $[20,7]$ in a framework in which analytical results can be obtained [23], and to analyze the statistics of the tumbling times, i.e. the time between two subsequent flips of the polymer [21,3,7]. The simplest model that reproduces qualitatively the behavior of polymers is the dumbbell model [22]. This model allows one to carry out some analytical calculations in the case which we analyze $[19,20,24,23]$, it is very easy and fast to simulate numerically (see Setion 2 and [25]) and reproduces qualitatively recent experimental results [3,7].

The paper is organized as follows: in Section 2, the evolution equation of the polymer and the numerical methods are briefly explained. Section 3 is devoted to the analysis of the statistics of thermal fluctuations of a flexible polymer placed into a linear shear flow. In our work we present the analysis of the stationary distribution of the polymer end-to-end vector and we study the distribution function of the polymer tumbling time, which can be measured experimentally. In Section 4, we study the angular dynamics of strongly elongated polymers, for which the size fluctuations are negligible. Finally, in Section 5, we study the elongation statistical properties of the end-to-end vector in a random flow with a large mean shear.

\section{Basic relations and numerical analysis}

We wish to analyze the behavior of a polymer in a generic simple shear flow experiencing the Langevin force [19]. In general there are two effects of the velocity field on a polymer molecule: the Lagrangian advection of the polymer and the elongation/relaxation dynamics due to velocity gradients. In all the cases discussed in this paper we disregard the Lagrangian dynamics by staying in the reference frame of the polymer center of mass. For the internal degrees of freedom of the polymer we use the simple dumbbell model [22], leaving the analysis of more realistic models for future studies [26]. In this case the basic equation describing the evolution of the polymer end-to-end vector $\boldsymbol{R}$ has the following form:

$$
\dot{R}_{i}=\sigma_{i j} R_{j}-\gamma R_{i}+\sqrt{\frac{2 \gamma R_{0}^{2}}{3}} \xi_{i}
$$

where $\sigma_{i j}=\partial_{j} v_{i}$ is the velocity gradient matrix, $\gamma$ the polymer relaxation rate, $\boldsymbol{\xi}$ the thermal noise term, which has white-noise statistics: $\left\langle\xi_{i}(t) \xi_{j}\left(t^{\prime}\right)\right\rangle=\delta_{i j} \delta\left(t-t^{\prime}\right)$, and $R_{0}$ is the equilibrium length in the absence of an external field. 
For incompressible flows there is no ambiguity in the discretization of the stochastic differential equation (1), and no additional contact term must be taken into account, so we resort to the Euler-Itô scheme without any loss of generality.

When the gradient $\sigma$ contains only a steady linear shear contribution we can write down the formal solution of (1) as:

$$
\dot{R}_{i}(t)=R_{i}(0) \mathrm{e}^{-\gamma t}+\mathrm{e}^{-\gamma t} \int_{0}^{t} \mathrm{e}^{\gamma t^{\prime}} s_{i j} R_{j}\left(t^{\prime}\right) \delta_{i x} \delta_{j y} \mathrm{~d} t^{\prime}-\gamma \mathrm{e}^{-\gamma t} \int_{0}^{t} \mathrm{e}^{\gamma t^{\prime}} R_{i}\left(t^{\prime}\right) \mathrm{d} t^{\prime}+\sqrt{\frac{2 \gamma R_{0}^{2}}{3}} \mathrm{e}^{-\gamma t} \int_{0}^{t} \mathrm{e}^{\gamma t^{\prime}} \xi_{i}\left(t^{\prime}\right) \mathrm{d} t^{\prime} .
$$

This equation can be discretized, and the terms containing the thermal noise can be rewritten as new gaussian variables with amplitudes that can be computed directly, so that the final solution reads:

$$
\begin{aligned}
R_{x}^{k+1}= & R_{x}^{k} \mathrm{e}^{-\gamma \Delta t}+\sqrt{\frac{R_{0}^{2}}{3}\left(1-\mathrm{e}^{-2 \gamma \Delta t}\right)} \eta_{x}^{k}+s \Delta t \mathrm{e}^{-\gamma \Delta t} R_{y}^{k}+s \frac{R_{0}}{\sqrt{3}} \eta_{y}^{k} \\
& \times \sqrt{\left[\frac{1}{2 \gamma^{2}}\left(1-\mathrm{e}^{-2 \gamma \Delta t}\right)-\frac{1}{\gamma} \Delta t \mathrm{e}^{-2 \gamma \Delta t}-\Delta t^{2} \mathrm{e}^{-2 \gamma \Delta t}\right]} \\
R_{i}^{k+1}= & R_{i}^{k} \mathrm{e}^{-\gamma \Delta t}+\sqrt{\frac{R_{0}^{2}}{3}\left(1-\mathrm{e}^{-2 \gamma \Delta t}\right)} \eta_{i}^{k}, \quad i=y, z
\end{aligned}
$$

where $\left\langle\eta_{i}^{k} \eta_{j}^{l}\right\rangle=\delta_{i j} \delta^{k l}$. The subscripts stand for the cartesian coordinates while the superscripts stand for the discretized time. As expected in the limit $\Delta t \rightarrow 0$ there is no contribution of $\eta_{y}$ in the first equation and the amplitudes are the same as in Eq. (1).

In the case of a random velocity gradient, $w_{i j}$, we have to generate the variables $w_{i j}^{k}$ such that they have the prescribed correlation function:

$$
\left\langle\sigma_{i j}(t) \sigma_{k l}\left(t^{\prime}\right)\right\rangle=D \delta\left(t-t^{\prime}\right)\left(4 \delta_{i k} \delta_{j l}-\delta_{i l} \delta_{k j}-\delta_{i j} \delta_{k l}\right),
$$

where again the Dirac delta function is substituted by a Kronecker symbol. In this case the modulus of the vector $\boldsymbol{R}$ grows indefinitely so that we can normalize it at each time step. In order to describe the elongation properties we can compute the maximum Lyapunov exponent $\lambda$ and the corresponding finite time Lyapunov exponent $\lambda_{T}$ [27].

\section{Thermally driven polymers}

In this section we will examine the case of a linear steady shear flow in the plane $X Y: \sigma_{i j}=s \delta_{i x} \delta_{j y}$, where $s$ is the shear rate. Eq. (1) has an explicit solution of the following form:

$$
R_{i}(t)=\exp (-\gamma t) W_{i j}(t) R_{j}(0)+\int_{0}^{t} \mathrm{~d} t^{\prime} \exp \left[-\gamma\left(t-t^{\prime}\right)\right] W_{i j}\left(t-t^{\prime}\right) \xi_{j}\left(t^{\prime}\right),
$$

where $W(t)=\exp (t \sigma)$. At large times the initial polymer elongation is forgotten and after averaging over the thermal fluctuations $\xi$ one can easily obtain the following distribution function:

$$
P(\boldsymbol{R})=(2 \pi)^{-3 / 2}(\operatorname{det} I)^{-1 / 2} \exp \left[-\frac{1}{2} \boldsymbol{R}^{\mathrm{T}} I^{-1} \boldsymbol{R}\right]
$$




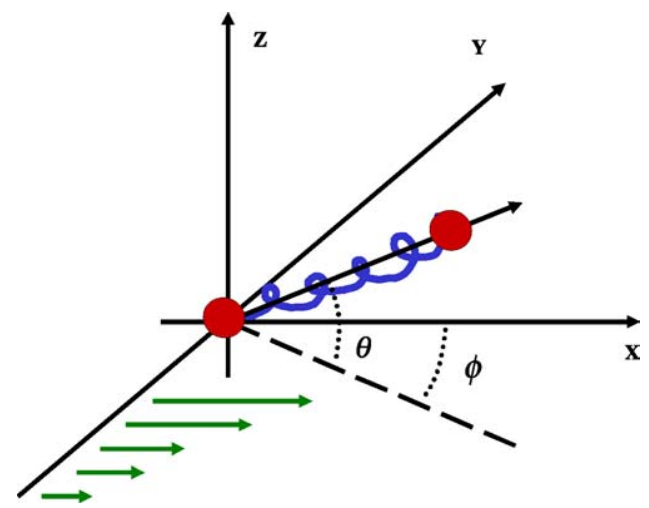

Fig. 1. Schematic figure explaining polymer orientation geometry.

$$
I=\frac{2 \gamma R_{0}^{2}}{3} \int_{0}^{\infty} \exp (-2 \gamma t) W(t) W^{\mathrm{T}}(t)
$$

The probability density function (PDF) (7) is valid for any velocity gradient. In the particular case of a steady shear flow in the $X Y$ plane, as shown in Fig. 1, the matrices can be found explicitly:

$$
W(t)=\left(\begin{array}{ccc}
1 & s t & 0 \\
0 & 1 & 0 \\
0 & 0 & 1
\end{array}\right), \quad I=\frac{R_{0}^{2}}{3}\left(\begin{array}{ccc}
1+\frac{s^{2}}{2 \gamma^{2}} & \frac{s}{2 \gamma} & 0 \\
\frac{s}{2 \gamma} & 1 & 0 \\
0 & 0 & 1
\end{array}\right)
$$

where the axis are sorted in the order $X-Z$. One can see that for large Weissenberg numbers $W i=s / \gamma \gg 1$, the mean polymer elongation in the $X$-direction is much larger than in transversal directions.

\subsection{Elongation PDF}

The PDF of elongation can be easily computed from (7): the elongation in the $y$ - and $z$-direction are independent of $W i$ and the two marginal PDFs are gaussian with zero mean value and variance $\left\langle R_{y}^{2}\right\rangle=\left\langle R_{z}^{2}\right\rangle=R_{0}^{2} / 3$, while in the mean flow direction the variance is $\left\langle R_{x}^{2}\right\rangle=\left(R_{0}^{2} / 3\right)\left(1+(1 / 2) W i^{2}\right)$ (Fig. 2).

The distribution function of $\boldsymbol{R}$ cannot be obtained analytically for an arbitrary value of $W i$, but one can study its behavior in two limiting cases. For $W i \ll 1$ the effect of the shear flow can be neglected, and we have the simple thermally driven polymer with linear relaxation force. The distribution function of its elongation has a simple gaussian form [22]:

$$
P(R)=\sqrt{\frac{2}{\pi}} \frac{R^{2}}{R_{0}^{3}} \exp \left[-\frac{R^{2}}{2 R_{0}^{2}}\right] .
$$

In the opposite case $W i \gg 1$ the system is strongly anisotropic and the main contribution to the polymer elongation comes from the $X$ component. This fact allows one to obtain the right tail of the elongation PDF. For $R \gg R_{0}$ one has

$$
P(R)=\sqrt{\frac{12}{\pi\left(2+W i^{2}\right)}} \frac{1}{R_{0}} \exp \left[-\frac{3 R^{2}}{\left(2+W i^{2}\right) R_{0}^{2}}\right] .
$$




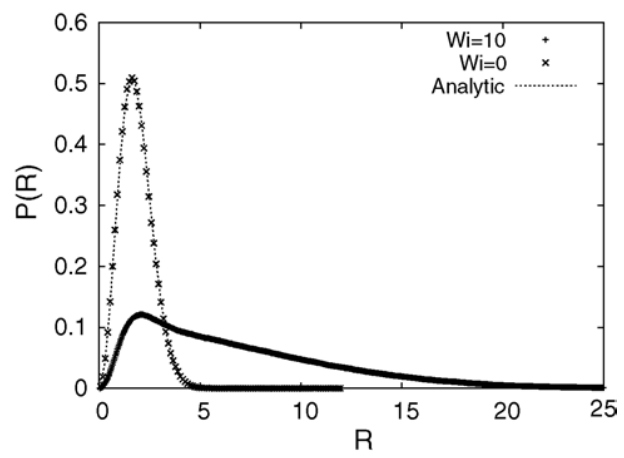

Fig. 2. The PDF of the modulus of the end-to-end vector $\boldsymbol{R}$ for different $W i$ numbers. While for $W i=0$ the PDF is concentrated around the radius of equilibrium, for higher values of $W i$ it is broader and the high elongation configurations become more and more probable.

\subsection{Angular $P D F$}

Next we are interested in the orientation of the polymer. The angular distribution function will allow us to show that for large $W i$ the polymer spends most of the time aligned to the $X$-axis, while for $W i \ll 1$ the orientation distribution is almost isotropic. In order to parameterize the orientation of the polymer we introduce the angles $\phi$ and $\theta$ as shown in Fig. 1 . The angle $\phi$ represents the deviation of the polymer end-to-end vector from the $X$-axis in the shear velocity plane $X Y$ while the angle $\theta$ gives us the polymer deviation in the transversal direction $Z$. Switching to spherical coordinates we have $R_{x}=R \cos \theta \cos \phi, R_{y}=R \cos \theta \sin \phi, R_{z}=R \sin \theta$. After integrating over the polymer elongation in Eq. (7) one immediately obtains the angular PDF:

$$
\mathcal{P}(\phi, \theta) \propto \frac{\cos \theta}{\left\{1-\left(\cos ^{2} \theta /\left(4+W i^{2}\right)\right)\left[W i^{2} \cos (2 \phi)+2 W i \sin (2 \phi)\right]\right\}^{3 / 2}} .
$$

The calculation of the two marginal PDFs is not possible in general, and we have to integrate Eq. (12) numerically (Figs. 3(a) and 4).

As one can see in Fig. 4, the PDF of $\theta$ decays as a power law (asymptotically $\mathcal{P}(\theta) \sim \theta^{-2}$ in the region $\phi_{t} \ll$ $\theta \ll 1$ ), and is symmetric about $\theta=0$. The higher the value of $W i$, the wider is the power law region.

In principle, if the polymer tumbles in the shear plane, we should consider a time-dependent PDF of $\phi$ on a unbounded domain. However, if we consider $\phi$ to be between 0 and $2 \pi$ one arrives at a stationary PDF, peaked in
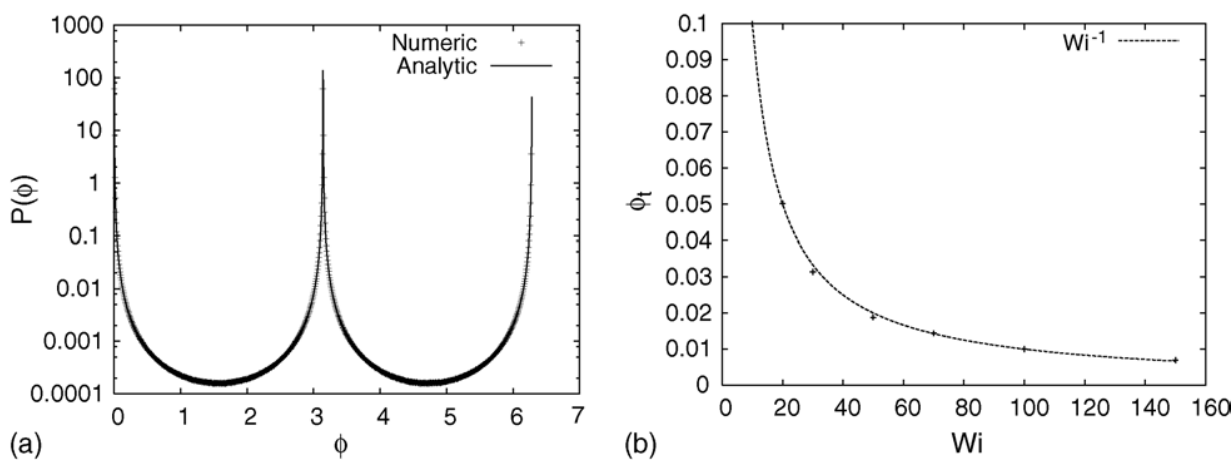

Fig. 3. The PDF of the angle $\phi$. (a) The analytic PDF is the numerical integration of (12), while the dots come from the numerical simulations. (b) The amplitude of the PDF's peak $\phi_{t}$ as a function of $W i$. 


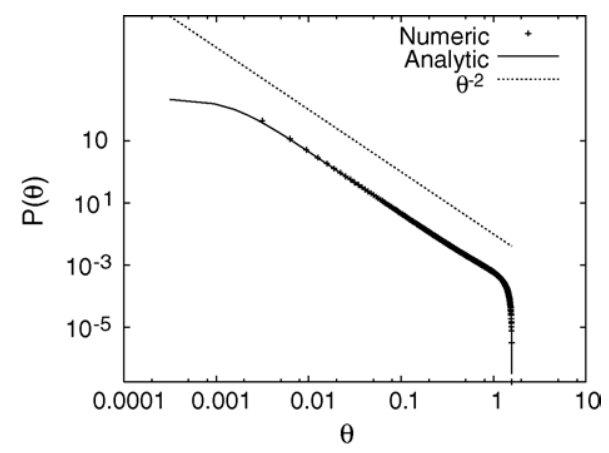

Fig. 4. The PDF of the angle $\theta$ is symmetric with respect to $\theta=0$ and can be described by a power law relationship over a wide range of values of $\theta$.

the regions $\phi=k \pi+\phi_{t}, k=0-2$ (Fig. 3(a)), where $\phi_{t} \sim 1 / W i$ as shown in Fig. 3(b). In the region where $\phi \gg \phi_{t}$ we have $\mathcal{P}(\phi) \sim \sin ^{-2} \phi$.

The angular PDFs tell us that any fluctuations in $\theta$ are not relevant to the tumbling dynamics, and that for the angle $\theta$ there is no relevant scale. The fact that increasing $W i$ the power law region becomes wider and wider means that when the shear is much stronger than the relaxation the polymer is nearly aligned with the $X$-axis. The PDF of the angle $\phi$ expresses the fact that the polymer spends most of the time in the vicinity of $\phi=0, \phi=\pi, \phi=2 \pi$. The polymer is aligned in the $X$-direction as a result of the shear flow, it fluctuates for a certain time in the region $\phi \sim \phi_{t}$, where the shape effects are strong. When thermally activated it goes beyond the region $\phi \gtrsim \phi_{t}$ and the shear becomes more important and induces a fast rotation of $\Delta \phi \simeq \pi$ or multiples thereof, that is the polymer tumbles.

\subsection{Tumbling time distributions}

In the previous two sections we have studied the stationary distribution functions which can be measured experimentally by averaging the polymer elongation and orientation over large time periods. However, as described in the introduction and in $[20,23]$ the dynamics of the polymer is non-stationary, due to continuous tumbling. The natural question which arises is whether there are some quantities, which would allow experimental observation and quantitative description of the tumbling process. In the recent papers by Chu et al. [3,5], the different time correlation functions of the polymer elongation were studied, and it has been shown that they can have different forms in the presence of shear. Here we introduce the tumbling time, which can be measured experimentally [7] and used to describe the tumbling process. As described above the most striking difference between the tumbling dynamics of the polymer in a shear flow and spinning in a rotational flow is the a-periodicity of the tumbling process. Due to the stochastic nature of tumbling, the tumbling time, i.e. time between subsequent flips of the polymer, is a random variable with relatively large fluctuations. Our task is now to study the distribution function of the tumbling time and to give some theoretical and numerical predictions of its dependence on the Weissenberg number (Fig. 5.).

In the case of the dumbbell model, we can define tumbling as a flip of the polymer induced by the thermal noise. When the polymer is in an unstable equilibrium configuration $\phi=k \pi, k=0-2$ the thermal noise can bring the polymer out of the region $\phi \sim \phi_{t}$, and this induces a fast rotation which takes a time of the order of $s^{-1}$. We define the tumbling time $\tau_{\phi}$ as the interval between two subsequent flips. This is typically the time between subsequent crossings of the lines $\phi=(k+1 / 2) \pi$. For $W i \gg 1$, as shown in the previous section (see Fig. 3(a)), the polymer spends only a small fraction of the total time far from the shear direction, and therefore the exact value of the angular threshold defining the tumbling time is not very important. Indeed this time is made up of two contributions: the time spent in the region $\phi \sim \phi_{t}$ and the duration of the rotation of order $s^{-1}$. The latter can be neglected for large Wi. 

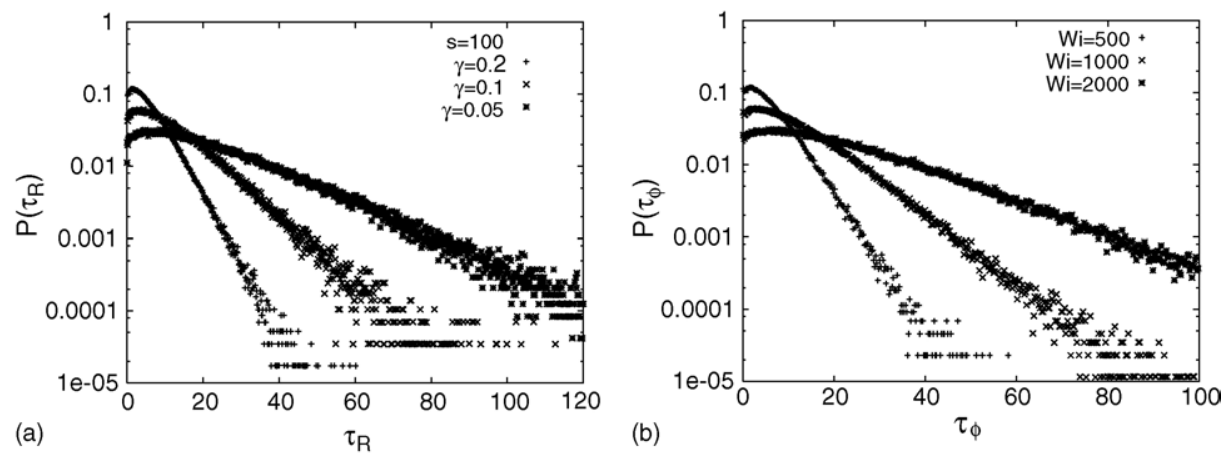

Fig. 5. The two different tumbling time PDFs. (a) The elongation-based tumbling time PDF. (b) The angle-based tumbling time PDF.

Experimental techniques do not always allow the polymer orientation angle $\phi$ to be resolved and therefore it is convenient to introduce another definition of the tumbling time $\left(\tau_{R}\right)$. This quantity measures the interval between subsequent changes between stretched and coiled polymer conformations. In other words, we start measuring when the length of the polymer exceeds a certain threshold value and we stop when it again becomes smaller than this threshold value.

While $\tau_{\phi}$ is the most natural definition it can be difficult to measure experimentally. On the other hand, the PDF of $\tau_{R}$ depends in a non-universal way on the threshold, but experimental techniques allows it to be measured when the polymer is sufficiently stretched.

In order to analyze the behavior of the tumbling time PDFs at large Weissenberg numbers we should analyze the dynamics of the polymer elongation projected onto the $X$-axis $\left(R_{x}\right)$. Indeed, the angle-based tumbling time $\tau_{\phi}$ measures the time intervals between subsequent events when $R_{x}=0$, while the elongation based tumbling time $\tau_{R}$ corresponds to the time intervals between crossings of a threshold value $R_{x}=R_{\mathrm{TH}}$. In order to find how the shape of the tumbling time PDF changes with increasing Wi number, we can rescale the time and the polymer size in the following way: $R_{x}=x R_{0} W i, R_{y}=y R_{0}, t=\gamma^{-1} \tau$, which leads to the following equations:

$$
\begin{aligned}
& \dot{x}=-x+y \\
& \dot{y}=-y+\sqrt{\frac{2}{3}} \zeta_{y} \\
& \left\langle\zeta_{y}(0) \zeta_{y}(\tau)\right\rangle=\delta(\tau) .
\end{aligned}
$$

In Eq. (13), we have omitted the term corresponding to $\xi_{x}$ because it is negligible in the large Weissenberg number regime compared to the other terms. Note that these equations do not depend on the Weissenberg number, and it enters the problem only through the threshold $R_{\mathrm{TH}}$. In our simulations we choose $R_{\mathrm{TH}}=\sqrt{3\left\langle R^{2}\right\rangle} \propto R_{0} W i$, so that in the dimensionless variables we have $x_{\mathrm{TH}}=1 / \sqrt{2}$ which does not depend on Weissenberg number either. From this analysis we conclude that the tumbling time PDF at large Weissenberg numbers approaches some universal form: its peak is positioned at characteristic time scale of order $\gamma^{-1}$, and as $\gamma$ increases the peak moves towards the origin. The problem of finding the exact form of this function is equivalent to the problem of finding the PDF of persistence times of a non-Markovian random process $x(\tau)$. This problem has recently attracted a lot of attention (see, e.g. ref. [28]), however no explicit solution is known for a general random process. Still it is possible to make some predictions on the PDF: the right tail of the PDF $\tau_{R}, \tau_{\phi} \gg \gamma^{-1}$ is exponential and has the form $P\left(\tau_{R}\right) \propto \exp \left[-c_{R} \gamma \tau_{R}\right], P\left(\tau_{\phi}\right) \propto \exp \left[-c_{\phi} \gamma \tau_{\phi}\right]$ because large tumbling times correspond to a large number $\gamma \tau \gg 1$ of unsuccessful attempts to cross the threshold. The correlation time of our stochastic process $R_{x}(t)$ is of order $\gamma^{-1}$, and therefore these attempts to cross the threshold are almost independent, and one should take the product of their probabilities, which leads to the above exponential laws. Both the PDFs of $\tau_{\phi}$ and $\tau_{R}$ are not well 
defined for $\tau_{\phi} \sim 0, \tau_{R} \sim 0$ since they are sensitive to brownian noise discretization: it is possible to tumble very rapidly when the polymer is coiled, and this is why the probability of measuring a tumbling time much smaller than $s^{-1}$ is not zero. Both the tumbling time definitions do not work very well in the case of small Wi experimentally because of the high resolution needed, and numerically because of the discretization procedure.

\section{Strongly elongated and rigid molecules}

Another physical situation we are interested in is the dynamics of strongly elongated polymers in random flows. In this model, described in detail in [20,24,23], the polymer is placed into a random flow above the coil-stretch transition, where the effect of the thermal fluctuations can be neglected. In this case the orientational dynamics of the polymer are decoupled from the evolution of the elongation, so that we can introduce the unit vector $n_{i}=R_{i} / R$, obeying the following evolution equation:

$$
\dot{n}_{i}=n_{j}\left(\delta_{i k}-n_{i} n_{k}\right) \nabla_{j} v_{k} .
$$

The velocity gradient consists of a regular shear part (as in the previous section), and an isotropic incompressible random part. The polymer size in the experiments is always much smaller than the characteristic viscous scale of the velocity field which allows us to assume smoothness of the velocity field. We will also assume short-correlated velocity field which corresponds to the so called Kraichnan-Batchelor model, which has been extensively studied in recent years [29]. In the framework of this model the velocity gradient matrix $\sigma_{i j}=\nabla_{j} v_{i}$ in the Lagrangian frame is described by a gaussian process with the following pair-correlation function [30]:

$$
\left\langle\sigma_{i j}(t) \sigma_{k l}\left(t^{\prime}\right)\right\rangle=D \delta\left(t-t^{\prime}\right)\left(4 \delta_{i k} \delta_{j l}-\delta_{i l} \delta_{k j}-\delta_{i j} \delta_{k l}\right),
$$

where $\sigma_{i j}$ is the gradient matrix of the random velocity component. Using the same notations as in the previous sections one arrives at the following dynamical equation for the angles:

$$
\begin{aligned}
& \dot{\phi}=-s \sin ^{2} \phi+\xi_{\phi}, \\
& \dot{\theta}=-s \frac{\sin (2 \phi)}{2} \sin \theta \cos \theta+\xi_{\theta},
\end{aligned}
$$

where $\xi_{\phi}$ and $\xi_{\theta}$ are zero mean random variables related to the fluctuating components of the velocity gradient. The statistics of both $\xi_{\phi}$ and $\xi_{\theta}$ can be obtained from the correlation function (17):

$$
\begin{aligned}
& \left\langle\xi_{\theta}(t) \xi_{\theta}\left(t^{\prime}\right)\right\rangle=4 D \delta\left(t-t^{\prime}\right) \\
& \left\langle\xi_{\phi}(t) \xi_{\phi}\left(t^{\prime}\right)\right\rangle=\frac{4 D}{\cos ^{2} \theta} \delta\left(t-t^{\prime}\right) .
\end{aligned}
$$

Note that the measure of configurations with $\theta \sim \pi / 2$ is small, thus making the formal singularity in Eq. (21) not essential. From Eq. (18) it turns out that the polymer experiences constant a-periodic tumbling in the $X Y$ plane $[20,24,23]$.

\subsection{Stationary angular PDF}

From a dimensional analysis it can be shown that for $s \ll D$ the characteristic values of the angles $\phi, \theta$ will be of $\operatorname{order}(D / s)^{1 / 3} \ll 1$. In this region one can set $\cos \theta=1$ in the expression (21) so that Eq. (18) becomes completely independent of $\theta$. Hence in this limit one derives:

$$
P_{\mathrm{st}}(\varphi)=\frac{\omega}{D} \int_{0}^{\infty} \mathrm{d} \phi \exp \left[-\frac{s}{8 D} \phi(\phi-2 \varphi)^{2}-\frac{s \phi^{3}}{24 D}\right]
$$




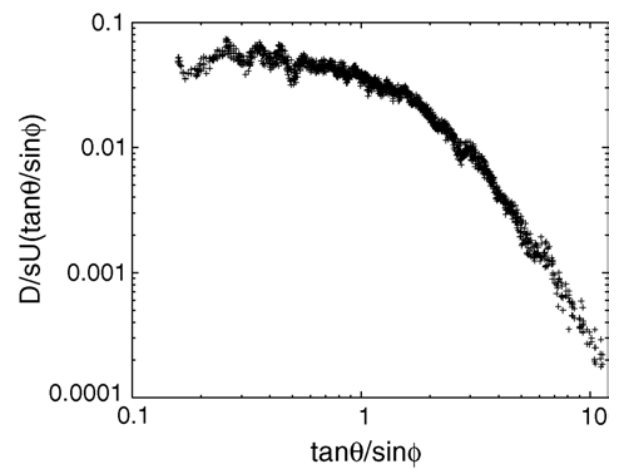

Fig. 6. The function $U(x)$, where $x=\tan \theta / \sin \phi$.

where $\omega$ is the mean rotation frequency of the polymer, which is determined from the normalization condition $\int_{0}^{\pi} P_{\mathrm{st}}(\phi) \mathrm{d} \phi=1$ and is given by

$$
\omega=\frac{\left(D s^{2}\right)^{1 / 3}}{4 \times 3^{1 / 6} \Gamma(7 / 6) \sqrt{\pi}} .
$$

The explicit form of the joint angular PDF is hard to compute analytically. However, one can obtain the expression for the tails of the PDF $\phi, \theta \gg(D / s)^{1 / 3}[24,19]$ :

$$
P(\phi, \theta)=\frac{U(\tan \theta / \sin \phi)}{\sin ^{3} \phi \cos ^{2} \theta},
$$

where $U(x)$ is an unknown function with an universal argument. Numerical simulations confirms this prediction, as shown in Fig. 6.

The two marginal PDFs obtained by numerical simulations show a behavior similar to that of thermally driven polymers. Also the phenomenology of the systems are similar, but now all the quantities depend on the ratio between the amplitude of the random velocity gradient and the mean shear. The PDF of the angle $\phi$ has peaks at $\phi \sim \phi_{t}+k \pi, k=0-2$, where $\phi_{t} \sim(D / s)^{1 / 3}$ and the PDF of $\theta$ has an algebraic core $\mathcal{P}(\theta) \sim \theta^{-2}$ for $(D / s) \ll 1$ (see Fig. 7).
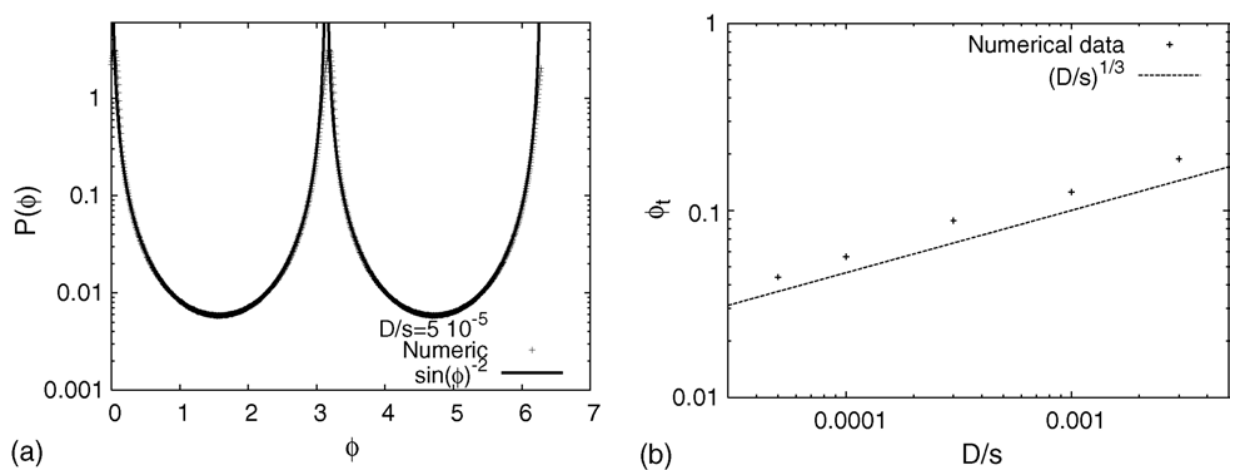

Fig. 7. The PDF of the angle $\phi$. (a) The agreement between the PDF and $\sin ^{-2} \phi$ is good in the region $\phi \gg \phi_{t}+k \pi, k=0-2$. (b) The behavior of $\phi_{t}$ as a function of $D / s$, with the dotted line shown as a guide only. 

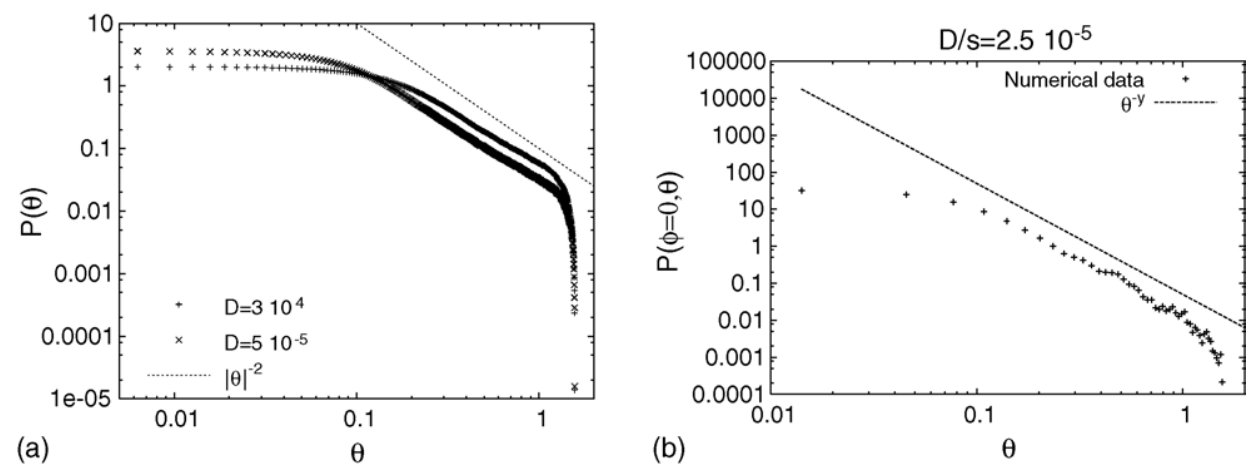

Fig. 8. The PDF of the angle $\theta$. (a) The PDF of the angle $\theta$ is symmetric with respect to $\theta=0$ and can be expressed by a power law in a wide range of $\theta$. (b) The power law for the core region $\phi \sim 0$. For this case $y \sim 3$.

In the stochastic region $|\phi| \lesssim \phi_{t}$ the tails of the $\theta$-angle PDF $P(\theta)$ are also algebraic $P(\phi=0, \theta) \propto \theta^{-y}$, but the exponent $y$ is non-universal and depends on the statistical properties of the random velocity gradient (see Fig. 8). It has been shown in [23] that a simple relation exists between the exponent $y$ and the entropy Cramèr function $S(x)$ of the Lyapunov exponent of the system (see Section 5). For the non-universal exponent $y$ the relation $y=S^{\prime}(x)$ holds, where $x$ is the solution of the equation $x S^{\prime}(x)=S(x)$. Numerical simulations show that in the case of an isotropic, short correlated, random velocity, the value of the exponent is approximately $y \approx 3$, as shown in Fig. 8 . This implies that it is subdominant on the background of the $\theta^{-2}$ tail coming from the regular dynamics region $|\phi| \gtrsim \phi_{t}$. However, in the general case of a finite-correlated and non-gaussian velocity field, one can imagine a situation where the non-universal exponent $y$ becomes smaller than the universal one $y<2$, and in this case the $\theta$-angle distribution becomes non-universal.

\subsection{Tumbling time distribution}

For rigid polymers with a fixed size the tumbling time can be defined only through the angular dynamics and therefore we will refer only to $\tau_{\phi}$. The evolution time of this system is $\tau_{t} \sim\left(s \phi_{t}\right)^{-1}$ so we expect that both the width and the maximum of the tumbling time distribution are $\tau_{t}$. The tails are related to the probability of passing (clockwise) the angle $\phi_{t}$ after a large amount of independent attempts, and can be estimated as $\mathcal{P}(\tau) \sim \mathrm{e}^{-E\left(D s^{2}\right)^{1 / 3} \tau}[20]$. In [23], it has been shown that the constant $E$ is connected with the ground state energy value of a one-dimensional quantum-mechanical system and can be estimated with simple numerical analysis $(E \simeq 0.45)$. It is possible to analyze the left tail of the tumbling time PDF:

$$
p(\tau) \propto \exp \left[-\frac{2 K^{4}(1 / 2)}{3 D s^{2} \tau^{3}}\right], \quad s^{-1} \ll \tau \ll\left(D s^{2}\right)^{-1 / 3},
$$

where $K(x)$ is an elliptical integral of the first kind, and also to give an exact expression for the tail $\tau \ll s^{-1}$ of the PDF. For large values of $s / D$ this tail can be barely observed experimentally or numerically, and the structure of the tail strongly depends on the statistics of the chaotic flow, and is therefore non-universal (see Fig. 9).

\section{Polymer elongation in chaotic flows}

In this final section we will study elongation statistics of the polymer in the case of random velocity plus mean shear. The polymer is not strongly elongated and can be treated as a linear dumbbell, as in Section 3. Such a situation 

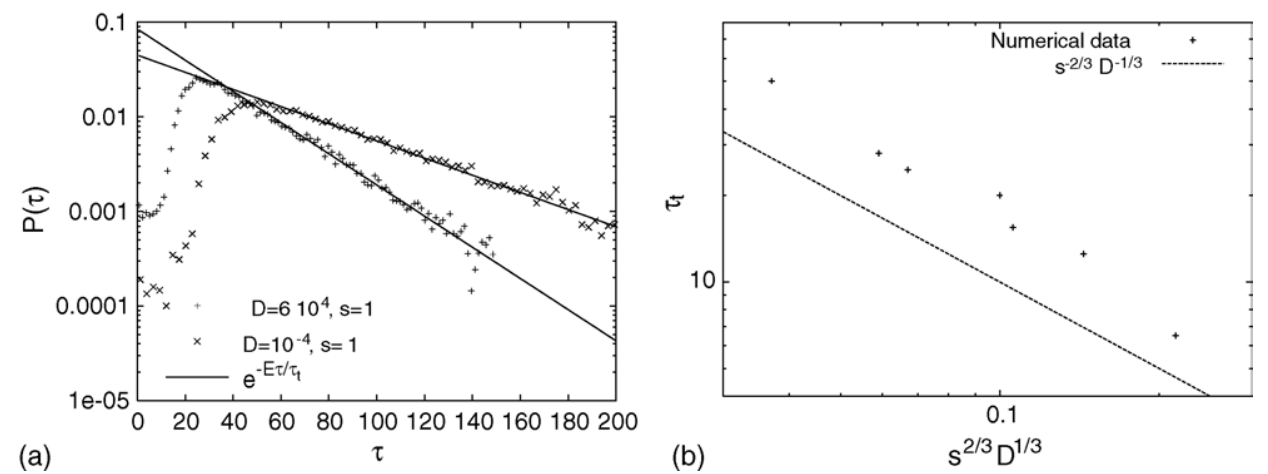

Fig. 9. The PDF of the tumbling time $\tau$. (a) The exponential tail of the PDF. (b) The behavior of $\tau_{t}$ as a function of $s^{2} D$.

corresponds to a flexible polymer in a chaotic flow below the coil-stretch transition[31]. Formally, this is the case when the maximum Lyapunov exponent is smaller than the inverse relaxation time $\lambda<\gamma$, where the Lyapunov exponent is the rate of divergence or convergence of two neighboring trajectories.

The equation governing the system is again Eq. (1) where the gradient of the velocity is decomposed into a regular shear part and a chaotic part. We can switch to spherical coordinates obtaining an evolution equation for the angles (as in Eqs. (18) and (19)) and an evolution equation for the modulus of the elongation vector [24,23]:

$$
\partial_{t} \ln R=-\gamma+\frac{1}{2} s \cos ^{2} \theta \sin 2 \phi+6 D+\eta,
$$

where in our model we assume $\left\langle\eta(t) \eta\left(t^{\prime}\right)\right\rangle=2 D \delta\left(t-t^{\prime}\right)$. Dimensional arguments show that the Lyapunov exponent should be proportional to $\left(D s^{2}\right)^{1 / 3}$ (see Fig. 10).

In this situation the polymer spends most of the time in the coiled state, but rare events lead to a large polymer stretching, when the flow becomes strong enough. It can be shown, that the right tails of polymer elongation PDF have the algebraic form $P(R) \propto R^{-1-q}$ [32,23], where the exponent $q$ depends on the parameters of the system. While in the thermal noise case the tails were Gaussian, here the probability of observing the polymer stretched is strongly enhanced. The algebraic behavior of the tail can be easily explained: the probability of having a local stretching rate $\lambda>\gamma$ for a large time $t$ decays exponentially with $t$, and during such events the polymer is stretched by a factor which grows exponentially with the time $t$.

It has been shown in $[32,23]$ that the exponent $q$ is related with the Cramèr function of the Lyapunov exponent of the flow [29]. This function can be found explicitly only in few cases, hence the main aim of this section is to

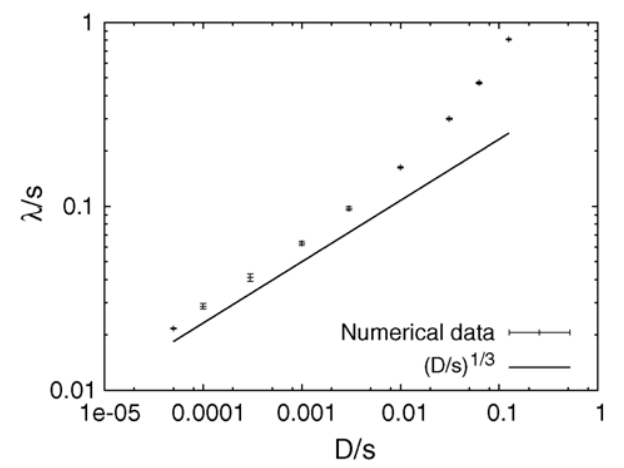

Fig. 10. The behavior of the Lyapunov exponent as a function of $D / s$. 


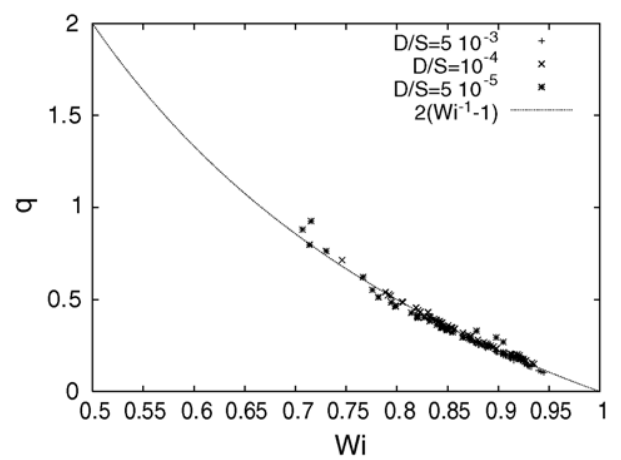

Fig. 11. The exponent of the elongation PDF as a function of Wi.

present numerical measurements of the Cramèr function and of the dependence of the exponent $q$ on the Weissenberg number $W i_{\lambda}=\lambda / \gamma$ below the coil-stretch transition (i.e. $W i_{\lambda}<1$ ). As shown in [23] the large deviation theory predicts that for large averaging times $T \gg\left(D s^{2}\right)^{-1 / 3} \equiv \tau_{T}$ the PDF of the Lyapunov exponent is:

$$
P\left(\lambda_{T}\right) \sim \exp \left(-\frac{T}{\tau_{T}} S\left(\lambda_{T} \tau_{T}\right)\right), \quad T \gg \tau_{T}
$$

where the function $S(x)$ is the Cramèr function. Note that for large but finite values of $T / \tau_{T}$ the measured Cramèr function $S_{T}(x)=-\left(\tau_{T} / T\right) \log P_{T}\left(x / \tau_{T}\right)$ depends on the time $T$, but the difference between $S_{T}(x)$ and $S(x)$ is significant only in the region $|x| \gtrsim T / \tau_{T} \gg 1$. Therefore in order to calculate the core of the Cramèr function we can use the finite time approximation. In our simulations we considered the ratio $T / \tau_{T}$ from 10 up to 280 .

In order to connect the Cramèr function with the exponent $q$ in the elongation PDF we need to use the Legendre transform (see refs. [32,23] for details):

$$
\begin{aligned}
& S(x)-x S^{\prime}(x)+\gamma \tau_{T} S^{\prime}(x)=0 \\
& q=S^{\prime}(x) .
\end{aligned}
$$

In $[32,33]$, the behavior of $q$ in the case of zero mean shear has been computed, and the calculations leads to:

$$
q=\frac{2}{\Delta}(\gamma-\lambda)
$$

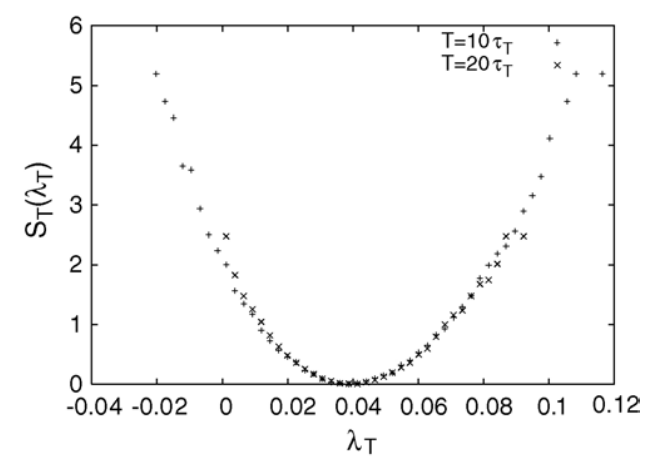

Fig. 12. The Cramèr function for two different times. 
where $\Delta$ is the variance of the Lyapunov exponent distribution. In the case of Kraichnan field without mean shear the ratio $\Delta$ is proportional to $\lambda$ (see ref. [29] for details), so that $q$ becomes proportional to $W i_{\lambda}^{-1}-1$. In [34], the case of shear turbulence has been analyzed and the scaling is the same as in Eq. (30).

The exponent $q$ extracted from numerical simulations is plotted in Fig. 11. The convergence of the Lyapunov exponent, given by the Cramèr function, is shown in Fig. 12.

\section{Conclusions}

The tumbling phenomenon $[21,3,20,7]$ has been studied in the framework of the linear dumbbell model, and some universal features of this motion are derived and numerically verified.

Three different examples of polymers in a linear, steady, plane, shear flow have been studied: (i) a flexible polymer experiencing thermal noise, (ii) a rigid polymer in a smooth random velocity gradient above the coilstretch transition $[31,32,6]$ ), and (iii) a flexible polymer in a smooth random velocity gradient below the coil-stretch transition.

In all three cases, the polymer tumbles aperiodically and the probability density function of the time between two successive tumbling events is exponential. While in the case of pure thermal noise, the typical tumbling time is determined only by the relaxation process, in the cases of random velocity gradient, the typical rate of divergence of two initially close lagrangian trajectories is the most important time scale.

The analysis of the statistics of orientation leads one to conclude that the phenomenology of these three situations is very similar: in the plane formed by the polymer end-to-end vector and the $Z$-axis there are no relevant scales and the polymer spends most of the time in the shear plane. In the shear plane, the dynamics is determined by the balance between the shear and the thermal fluctuations. The majority of time is spent nearly aligned to the velocity field. Aperiodically the shear induces a tumbling event.

The elongation dynamics is determined only by the balance between the relaxation and the stretching due to the shear flow in the thermal noise case. In the other two cases stretching is determined by the presence of a positive Lyapunov exponent. In the case below the coil-stretch transition the tail of the PDF can be determined by measuring the statistics of the Lyapunov exponent.

\section{Acknowledgements}

We would like to thank A. Celani, M. Chertkov, I. Kolokolov and V. Lebedev for many fruitful and inspiring discussions and S. Geraschenko and V. Steinberg for many useful comments and interest in our work. We are grateful to Chris Carr and Colm Connaughton who helped us to develop the final form of this paper. We acknowledge hospitality of CNLS at Los Alamos National Laboratory where this work was partially done. KT was supported by RFBR grant 04-02-16520a and INTAS grant Nr 04-83-2922.

\section{References}

[1] T.T. Perkins, D.E. Smith, S. Chu, Single polymer dynamics in an elongational flow, Science 2762016 (1997).

[2] D.E. Smith, S. Chu, Response of flexible polymers to a sudden elongational flow, Science 281 (1998) 1335.

[3] D.E. Smith, H.P. Babcock, S. Chu, Single polymer dynamics in steady shear flow, Science 283 (1999) 1724.

[4] J.S. Hur, E.S.G. Shaqfeh, R.G. Larson, Brownian dynamics simulations of single DNA molecules in shear flow, J. Rheol. 44 (2000) 713.

[5] J.S. Hur, E. Shaqfeh, H.P. Babcock, D.E. Smith, S. Chu, Dynamics of dilute and semidilute DNA solutions in the start-up of shear flow, J. Rheol 45 (2001) 421.

[6] S. Gerashchenko, C. Chevallard, V. Steinberg, Single polymer dynamics: coil-stretch transition in a random flow, Europhys. Lett. 71 (2) (2005) 221-227. 
[7] S. Gerashchenko and V. Steinberg, Statistics of tumbling of a single polymer molecule in shear flow, Phys. Rev. Lett., submitted for publication (see also nlin.CD/0503028).

[8] R.E. Teixeira, H.P. Babcock, E. Shaqfeh, S. Chu, Shear thinning and tumbling dynamics of single polymers in the flow-gradient plane, Macromolecules 38 (2005) 581-592.

[9] C.M. Schroeder, R.E. Teixeira, E.S.G. Shaqfeh, S. Chu, Dynamics of DNA in the flow-gradient plane of steady shear flow: observations and simulations, Macromolecules 38 (2005) 1967-1978.

[10] B. Ladoux, J. Quivy, P.S. Doyle, G. Amouzni, J. Viovy, Direct imaging of singlemolecules: from dynamics of a single DNA chain to the study of complex DNA-protein interactions, Sci. Prog. 84 (4) (2001) 267.

[11] S. Chu, Biology and polymer physics at the single-molecule level, Phil. Trans. R. Soc. Lond. A $361689-6986$ (2003).

[12] S. Manneville, P. Cluzel, J.L. Viovy, D. Chatenay, F. Caron, Evidence for the universal scaling behaviour of a freely relaxing DNA molecule, Europhys. Lett. 36 (1996) 413.

[13] R.G. Larson, T.T. Perkins, D.E. Smith, S. Chu, Hydrodynamics of a DNA molecule in a flow field, Phys. Rev. E 55 (1997) 1794.

[14] Y. Cui, C. Bustamante, Pulling a single chromatin fiber reveals the forces that maintain its higher-order structure, Proc. Natl. Acad. Sci. 97 (2000) 127.

[15] M. Hegner, S.B. Smith, C. Bustamante, Polymerisation and mechanical properties of single RecA-DNA filaments, Proc. Natl. Acad. Sci. 96 (1999) 10109.

[16] H. Yin, M.D. Wang, K. Svoboda, R. Landick, S.M. Block, J. Gelles, Transcription against an applied force, Science 270 (1995) 1653.

[17] G.J. Wuite, S.B. Smith, M. Young, D. Keller, C. Bustamante, Single molecule studies of the effect of template tension on T7 DNA polymerase activity, Nature 404 (2000) 103.

[18] A. Groisman, V. Steinberg, Elastic turbulence in curvilinear flows of polymer solutions, New J. Phys. 6 (2004) 29.

[19] E.J. Hinch, L.G. Leal, The effect of Brownian motion on the rheological properties of a suspension of non-spherical particles, J. Fluid Mech. 52 (1972) 634.

[20] M. Chertkov, I. Kolokolov, V. Lebedev and K. Turitsyn, Tumbling of polymers in random flow with mean shear, J. Fluid Mech., submitted for publication.

[21] T.W. Liu, Flexible polymer chain dynamics and rheological properties in steady flows, J. Chem. Phys. 90 (1989) 5826.

[22] R.B. Bird, C.F. Curtiss, R.C. Armstrong, O. Hassager, Dynamics of Polymeric Liquids, Wiley, New York, 1987.

[23] K. Turitsyn, Polymer dynamics in chaotic flows with strong shear component, Submitted to Phys. Rev. E (see also nlin.CD/0501025).

[24] M. Chertkov, I. Kolokolov, V. Lebedev, K. Turitsyn, Polymer statistics in a random flow with mean shear, J. Fluid Mech. 531 (2005) $251-260$

[25] H.C. Ottinger, Stochastic Processes in Polymeric Liquids, Springer, Berlin, 1995.

[26] A. Celani, A. Puliafito, K. Turitsyn, Polymers in linear shear flow: a numerical study, EuroPhys. Lett. 70 (2005) 464.

[27] G. Benettin, L. Galgani, A. Giorgilli, J.-M. Strelcyn, Lyapunov exponents for smooth dynamical systems and for Hamiltonian systems; a method for computing all of them, Meccanica 15 (1980) 9-30.

[28] S.N. Majumdar, C. Sire, Survival probability of a Gaussian non-Markovian process: application to the $T=0$ dynamics of the Ising model, Phys. Rev. Lett. 77 (1996) 1420.

[29] G. Falkovich, C. Gawedzky, M. Vergassola, Particles and fields in fluid turbulence, Rev. Mod. Phys. 73 (4) (2001) $913-975$.

[30] R.H. Kraichnan, Small-scale structure of a scalar field convected by turbulence, Phys. Fluids 11 (1968) 945-963.

[31] P.G. De Gennes, Coil-stretch-transition of dilute felxible polymersunder ultra-high velocity gradients, J. Chem Phys 60 (1974) 5030.

[32] E. Balkovsky, A. Fouxon, V. Lebedev, Turbulent dynamics of polymer solutions, Phys. Rev. Lett. 84 (2000) 4765;

E. Balkovsky, A. Fouxon, V. Lebedev, Turbulent dynamics of polymer solutions, Phys. Rev. E 64 (2001) 056301.

[33] A. Celani, S. Musacchio, D. Vincenzi, Polymer transport in random flow, J. Stat. Phys. 118 (2005) 529-552.

[34] B. Eckhardt, J. Kronjager, J. Schumacher, Stretching of polymers in a turbulent environment, Comp. Phys. Commun. 147 (2002) 538. 